\title{
Disambiguation of biomedical text using diverse sources of information
}

\author{
Mark Stevenson*1, Yikun Guo ${ }^{1}$, Robert Gaizauskas ${ }^{1}$ and David Martinez ${ }^{2}$
}

Address: ${ }^{1}$ Department of Computer Science, University of Sheffield, Regent Court, 211 Portobello, Sheffield, S1 4DP, UK and ${ }^{2}$ NICTA Victoria and Department of Computer Science, Software Engineering, University of Melbourne, Victoria 3010, Australia

Email: Mark Stevenson* - m.stevenson@dcs.shef.ac.uk; Yikun Guo - g.yikun@dcs.shef.ac.uk; Robert Gaizauskas - r.gaizauskas@dcs.shef.ac.uk; David Martinez - davidm@csse.unimelb.edu.au

* Corresponding author

from Natural Language Processing in Biomedicine (BioNLP) ACL Workshop 2008

Columbus, OH, USA. 19 June 2008

Published: 19 November 2008

BMC Bioinformatics 2008, 9(SuppI II):S7 doi:I0.1186/I47I-2105-9-SII-S7

This article is available from: http://www.biomedcentral.com/I47I-2I05/9/SI I/S7

(c) 2008 Stevenson et al; licensee BioMed Central Ltd.

This is an open access article distributed under the terms of the Creative Commons Attribution License (http://creativecommons.org/licenses/by/2.0),

which permits unrestricted use, distribution, and reproduction in any medium, provided the original work is properly cited.

\begin{abstract}
Background: Like text in other domains, biomedical documents contain a range of terms with more than one possible meaning. These ambiguities form a significant obstacle to the automatic processing of biomedical texts. Previous approaches to resolving this problem have made use of various sources of information including linguistic features of the context in which the ambiguous term is used and domain-specific resources, such as UMLS.
\end{abstract}

Materials and methods: We compare various sources of information including ones which have been previously used and a novel one: $\mathrm{MeSH}$ terms. Evaluation is carried out using a standard test set (the NLM-WSD corpus).

Results: The best performance is obtained using a combination of linguistic features and MeSH terms. Performance of our system exceeds previously published results for systems evaluated using the same data set.

Conclusion: Disambiguation of biomedical terms benefits from the use of information from a variety of sources. In particular, MeSH terms have proved to be useful and should be used if available.

\section{Background}

The number of documents discussing biomedical science is growing at an ever increasing rate, making it difficult to keep track of recent developments. Automated methods for cataloging, searching and navigating these documents would be of great benefit to researchers working in this area, as well as having potential benefits to medicine and other branches of science. Lexical ambiguity, the linguistic phenomenon where a term (word or phrase) has more than one potential meaning, makes the automatic processing of text difficult. For example, "cold" has several possible meanings in the Unified Medical Language System (UMLS) Metathesaurus [1] including "common cold", "cold sensation" and "Chronic Obstructive Airway 
Disease (COLD)". Weeber et al. [2] analysed MEDLINE abstracts and found that $11.7 \%$ of phrases were ambiguous relative to the UMLS Metathesaurus.

The ability to accurately identify the meanings of terms is an important step in automatic text processing. It is necessary for applications such as information extraction and text mining which are important in the biomedical domain for tasks such as automated knowledge discovery. The NLM Indexing Initiative [3] attempted to automatically index biomedical journals with concepts from the UMLS Metathesaurus and concluded that lexical ambiguity was the biggest challenge in the automation of the indexing process. Friedman [4] reported that an information extraction system originally designed to process radiology reports had problems with ambiguity when it was applied to more general biomedical texts. During the development of an automated knowledge discovery system Weeber et al. [5] found that is was necessary to resolve the ambiguity in the abbreviation MG (which can mean 'magnesium' or 'milligram') in order to replicate a wellknown literature-based discovery concerning the role of magnesium deficiency in migraine headaches [6].

Word Sense Disambiguation (WSD) is the process of resolving lexical ambiguities. WSD has been actively researched since the 1950s and is regarded as an important part of the process of understanding natural language texts. A comprehensive description of current work in WSD is beyond the scope of this paper although overviews may be found in $[7,8]$. Schuemie et al. [9] provide an overview of WSD in the biomedical domain. Previous researchers have used a variety of approaches for WSD of biomedical text. Some of them have taken techniques proven to be effective for WSD of general text and applied them to ambiguities in the biomedical domain, while others have created systems using domain-specific biomedical resources. However, there has been no direct comparison of which information sources are the most useful or whether combining a variety sources, a strategy which has been shown to be successful for WSD in the general domain $[10,11]$, also improves results in the biomedical domain.

This paper compares the effectiveness of a variety of information sources for WSD in the biomedical domain. These include features which have been commonly used for WSD of general text as well as information derived from domain-specific resources, including MeSH terms.

The remainder of this section provides an overview of various approaches to WSD in the biomedical domain. The Methods section outlines our approach, paying particular attention to the various types of information used by our system. An evaluation of this system is presented in the
Results section, the implications of which can be found in the Discussion section.

\section{The NLM-WSD data set}

Research on WSD for general text in the last decade has been driven by the SemEval frameworks http://www.sen seval.org which provide a set of standard materials for a variety of semantic evaluation tasks [12]. At this point there is no specific collection for the biomedical domain in SemEval, but a test collection for WSD in biomedicine, the NLM-WSD data set [2], is used as a benchmark by many independent groups. (An alternative collection is described by Widdows et al. [13], although the authors acknowledge that the low levels of inter-annotator agreement for the sense tags make the use of this data problematic.) The Unified Medical Language System (UMLS) Metathesaurus was used to define the set of possible meanings in the NLM-WSD data set. In UMLS strings are mapped onto concepts, indicating their meaning. Strings which map onto more than one concept are ambiguous. For example, the string "culture" maps onto the concepts 'Anthropological Culture' (e.g. "The aim of this paper is to describe the origins, initial steps and strategy, current progress and main accomplishments of introducing a quality management culture within the healthcare system in Poland.") and 'Laboratory Culture' (e.g. "In peripheral blood mononuclear cell culture streptococcal erythrogenic toxins are able to stimulate tryptophan degradation in humans"). 50 terms which are ambiguous in UMLS and occur frequently in MEDLINE were chosen for the NLMWSD data set. 100 instances of each term were selected from citations added to the MEDLINE database in 1998 and manually disambiguated by 11 annotators. Twelve terms were flagged as "problematic" due to substantial disagreement between the annotators. There are an average of 2.64 possible meanings per ambiguous term and the most ambiguous term, "cold", has five possible meanings. Concepts which were judged to be very similar in meaning were merged. For example, two concepts for "depression": 'Depressive episode, unspecified' and 'Mental Depression'. In addition to the meanings defined in UMLS, annotators had the option of assigning a special tag ("none") when none of the meanings in UMLS were judged to be appropriate.

Various researchers have chosen to evaluate their systems against subsets of this data set. Liu et al. [14] used a set of 22 terms, saying "We excluded 12 [terms] that Weeber et al. considered problematic, as well as 16 terms in which the majority sense occurred with over $90 \%$ of instances." However, the 22 terms used to evaluate their system include "mosaic" and "nutrition" which Weeber et al. [2] flagged as problematic. Leroy and Rindflesch [15] used a set of 15 terms for which the majority sense accounted for less than $65 \%$ of the instances. Joshi et al. [16] evaluated 
against the set union of those two sets, providing 28 ambiguous terms. McInnes et al. [17] used the set intersection of the two sets (dubbed the "common subset") which contained 9 terms. The terms that form these various subsets are shown in Figure 1.

The 50 terms which form the NLM-WSD data set represent a range of challenges for WSD systems. The Most Frequent Sense (MFS) heuristic has become a standard baseline in WSD [18] and is simply the accuracy which would be obtained by assigning the most common meaning of a term to all of its instances in a corpus. Despite its simplicity, the MFS heuristic is a hard baseline to beat, particularly for unsupervised systems, because it uses handtagged data to determine which sense is the most frequent. Analysis of the NLM-WSD data set showed that the MFS over all 50 ambiguous terms is $78 \%$. The different subsets have lower MFS, indicating that the terms they contain are more difficult to disambiguate. The 22 terms used by Liu et al. [14] have an MFS of $69.9 \%$ while the set used by Leroy and Rindflesch [15] has an MFS of 55.3\%.
The union and intersection of these sets have MFS of $66.9 \%$ and $54.9 \%$ respectively.

\section{WSD of biomedical text}

A standard approach to WSD is to make use of supervised machine learning systems which are trained on examples of ambiguous words in context along with the correct sense for that usage. The models created are then applied to new examples of that word to determine the sense being used.

Approaches which are adapted from WSD of general text include [14]. Their technique uses a supervised learning algorithm with a variety of features consisting of a range of collocations of the ambiguous word and all words in the abstract. They compared different supervised machine learning algorithms and found that a decision list worked best. Their best system correctly disambiguated $78 \%$ of the occurrences of 22 ambiguous terms in the NLM-WSD data set (see Figure 1).

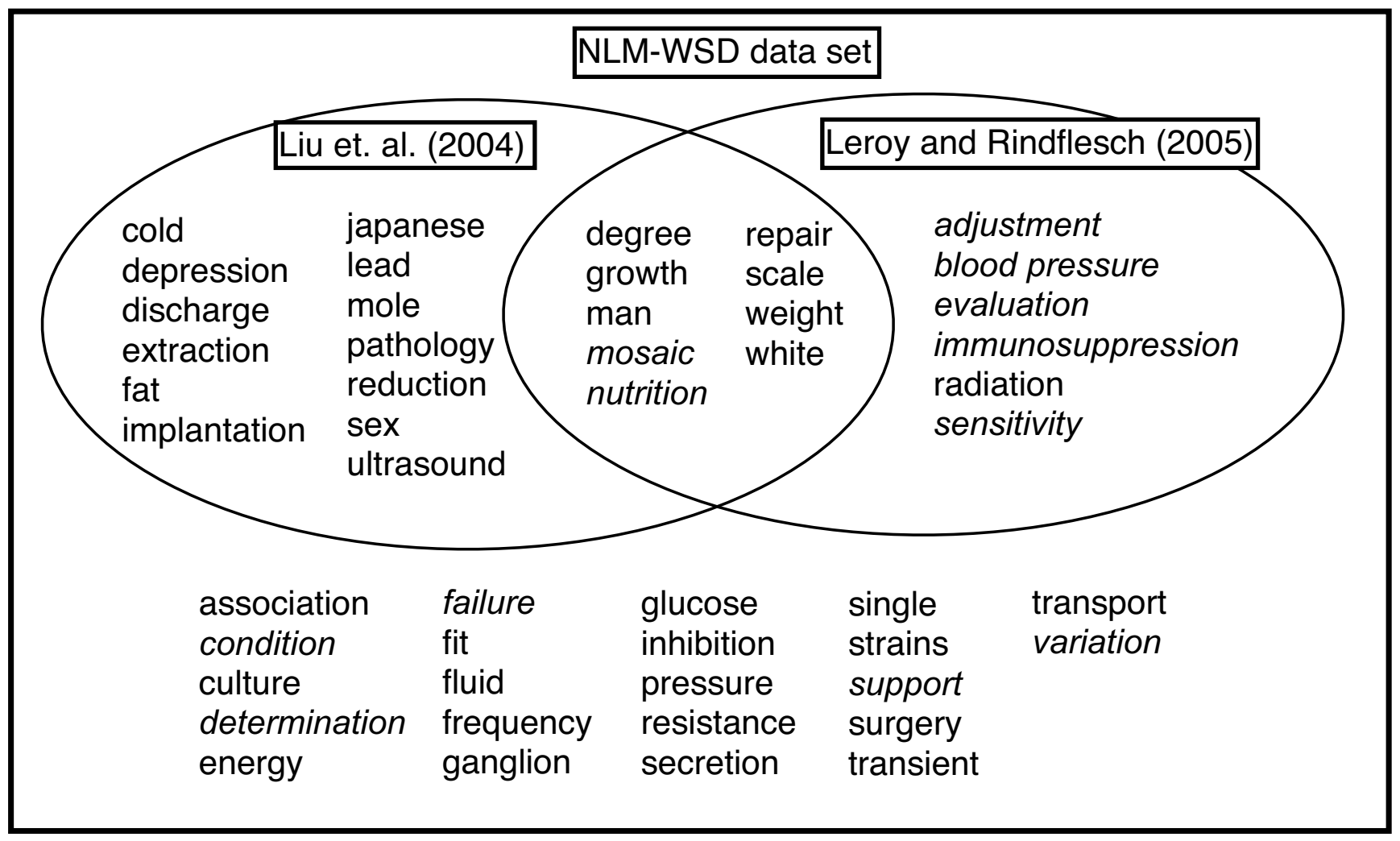

Figure I

The NLM-WSD test set and some of its subsets. The 12 terms which Weeber et al. [2] described as "problematic" due to low levels of agreement between annotators are shown in italics. The test set used by Joshi et al. [16] comprises the set union of the terms used by Liu et al. [14] and Leroy and Rindflesch [15] while the "common subset" is formed from their intersection. 
Joshi et al. [16] also use collocations as features and experimented with five supervised learning algorithms: Support Vector Machines, Naive Bayes, decision trees, decision lists and boosting. The Support Vector Machine performed best scoring $82.5 \%$ on a set of 28 words (see Figure 1) and $84.9 \%$ on the 22 terms used by Liu et al. [14]. Performance of the Naive Bayes classifier was comparable to the Support Vector Machine, while the other algorithms were hampered by the large number of features.

Examples of approaches which have made use of knowledge sources specific to the biomedical domain include Leroy and Rindflesch [15] who used information from the UMLS Metathesaurus. They used the MetaMap tool [19] which identifies the relevant UMLS concepts for a piece of text. Leroy and Rindflesch used knowledge about whether the ambiguous word is the head word of a phrase identified by MetaMap, the ambiguous word's part of speech, semantic relations between the ambiguous words and surrounding words from UMLS as well as semantic types of the ambiguous word and surrounding words. Naive Bayes was used as a learning algorithm. This approach correctly disambiguated $65.5 \%$ of word instances from a set of 15 terms (see Figure 1). Humphrey et al. [20] presented an unsupervised system that also used semantic types from UMLS. They constructed semantic type vectors for each word from a large collection of MEDLINE abstracts. This allowed their method to perform disambiguation at a coarser level, without the need for labeled training examples. In most cases the semantic types can be mapped to the UMLS concepts used to annotate instances in the NLM-WSD corpus but not for all terms. In addition, this approach could not disambiguate instances which had been annotated with the "none" tag which indicated that none of the meanings in UMLS were judged to be appropriate. Five terms were excluded from their evaluation, four ("cold", "man", "sex" and "weight") because the semantic types could not be mapped onto UMLS concepts and the other ("association") because all instances of that term were assigned the "none" tag. In addition, only $67 \%$ of the instances for the remaining 45 terms were used for evaluation and, since instances with the "none" tag were also excluded, their system was only evaluated against an average of $54 \%$ of the instances of these terms. An accuracy of $78.6 \%$ was reported across these instances. McInnes et al. [17] also made use of information provided by MetaMap. In UMLS each concept has a Concept Unique Identifier (CUI) and these are also assigned by MetaMap. The information contained in CUIs is more specific than in the semantic types applied by Leroy and Rindflesch [15] and Humphrey et al. [20]. For example, two of the CUIs for the term "cold" in UMLS, "C0205939: Common Cold" and "C0024117: Chronic Obstructive Airway Disease", share the same semantic type: "Disease or Syndrome". McInnes et al. [17] were interested in exploring whether the more specific information contained in CUIs was more effective than UMLS semantic types. Their best result was reported for a system which represented each sense by all CUIs which occurred at least twice in the abstract surrounding the ambiguous word. They used a Naive Bayes classifier as the learning algorithm and reported an accuracy of $74.5 \%$ on the set of ambiguous terms tested by Leroy and Rindflesch [15] and $80.0 \%$ on the set used by Joshi et al. [16]. They concluded that CUIs are more useful for WSD than UMLS semantic types but that they are not as robust as features which are known to work in general English, such as unigrams and bigrams. Unfortunately, direct comparison of the various WSD systems which have been evaluated on the NLMWSD data set is not straightforward. Firstly, as we have described, systems have been tested against a variety of ambiguous terms. A more subtle problem arises in the way in which researchers have chosen to present their results. With the exception of unsupervised systems $[15,20]$, which do not require training data, all approaches involve training a classifier using some portion of the available data and then testing against the remaining unseen portion. These supervised approaches normally involve choices over how to set the parameters which define the group of features used. For example, Liu et al. [14] compared a total of 22 different feature sets by varying the size of the context window around the ambiguous word and the terms which are extracted. One approach $[14,16]$ is to experiment with a variety of parameters and choose the best one for each ambiguous term. For example, the $78 \%$ accuracy figure quoted by Liu et al. [14] is obtained by choosing the result from the best classifier for each of the 22 terms used in their evaluation. We refer to this as per-term parameter setting. An alternative methodology involves applying the same parameters to all terms. For example, the results reported by McInnes et al. [17] are obtained by using the same parameters for all terms rather than selecting the best result for each. We call this global parameter setting.

It would be preferable to automate the process of parameter setting as far as possible however this would be difficult for per-term parameter setting, particularly for a data set such as NLM-WSD where there are only 100 instances for each ambiguous term and many senses with occur only a few times. The alternative approach, global parameter setting, is less affected by this problem and has the advantage that the settings are more likely to be suitable for terms other than the ones which are contained in the test collection. The global parameter setting methodology is used in the experiments described later in this paper. 


\section{Methods}

Our approach is to adapt a state-of-the-art WSD system to the biomedical domain by augmenting it with additional domain-specific and domain-independent information sources. Our basic system [21] participated in the Senseval-3 challenge [22] with a performance close to the best system for both the English and Basque lexical sample tasks. The method is based on a supervised learning approach and uses features derived from text around the ambiguous word which are domain independent. We refer to these as linguistic features. This feature set has been adapted for the disambiguation of biomedical text by adding further linguistic features and two different types of domain-specific features: CUIs (as used by McInnes et al. [17]) and Medical Subject Heading (MeSH) terms.

\section{Features}

Our feature set contains a number of parameters which were set empirically (e.g. threshold for unigram frequency in the linguistic features). In addition, we use the entire abstract as the context of the ambiguous term for relevant features rather than just the sentence containing the term. Effects of varying these parameters are similar to results reported in previous work [14,16,17], for example using the entire abstract as context yields more accurate results than using only the sentence containing the ambiguous term. Since these results are not novel we do not report them in this paper.

\section{Linguistic features}

The system uses a wide range of domain-independent features which are commonly used for WSD.

- Local collocations: A total of 41 features which extensively describe the context of the ambiguous word and fall into two main types: (1) bigrams and trigrams containing the ambiguous word constructed from lemmas, word forms or PoS tags (assigned using maximum-entropybased part of speech tagger [23]) and (2) preceding/following lemma/word-form of the content words (adjective, adverb, noun and verb) in the same sentence with the target word. For example, consider the sentence below with the target word adjustment.

"Body surface area adjustments of initial heparin dosing..."

The features would include the following: left-contentword-lemma "area adjustment", right-function-wordlemma "adjustment of ", left-POS "NN NNS", right-POS "NNS IN", left-content-word-form "area adjustments", right-function-word-form "adjustment of ", etc.

- Salient bigrams: Salient bigrams within the abstract with high log-likelihood scores computed from the NLM-WSD corpus, as described by Pedersen [24]. In the experiments, bigrams that occur more than once and have a log-likelihood higher than 6.635 are included as features.

- Unigrams: Lemmas of all content words (nouns, verbs, adjectives, adverbs) in the target word's sentence and, as a separate feature, lemmas of all content words within $\mathrm{a} \pm$ 4-word window around the target word, excluding those in a list of corpus-specific stopwords (e.g. "ABSTRACT", "CONCLUSION"). In addition, the lemmas of any unigrams which appear at least twice in the entire corpus and are found in the abstract are also included as features. This feature was not used by [21], but Joshi et al. [16] found it to be useful for this task.

A previous version of our system [25] included syntactic dependencies, such as subject and noun-modifier of ambiguous terms, as an additional feature. These features were also used by Agirre and Martinez [21] and were extracted by a set of manually-created heuristics applied to part of speech tagged text. However, we found that removing these features led to a small increase in performance. The likely reason for this is that these features are noisy since the dependencies are difficult to identify accurately. In addition the heuristics used were not developed to be applied on biomedical documents.

\section{Concept Unique Identifiers (CUIs)}

We follow the approach presented by McInnes et al. [17] to generate features based on UMLS Concept Unique Identifiers (CUIs). The MetaMap program [19] identifies all words and terms in a text which could be mapped onto a UMLS CUI. MetaMap does not disambiguate the senses of the concepts; instead it enumerates all the possible combinations of the concept names found. For example, MetaMap will segment the phrase "Body surface area adjustments of initial heparin dosing ..." into two chunks: "Body surface area adjustments" and "of initial heparin dosing". The first chunk will be mapped onto four CUIs, two with the concept name "Body Surface Area": "C0005902: Diagnostic Procedure" and "C1261466: Organism Attribute" and a further pair with the name "Adjustments": "C0456081: Health Care Activity" and "C0871291: Individual Adjustment". CUIs which occur more than three times in the abstract containing the ambiguous word are included as features.

\section{Medical Subject Headings (MeSH)}

The final feature is also specific to the biomedical domain. Medical Subject Headings (MeSH) [26] is a controlled vocabulary for indexing biomedical and health-related information and documents. MeSH terms are manually assigned to abstracts by human indexers. The latest version of MeSH contains over 24,000 terms organised into an 11-level hierarchy. The terms assigned to the abstract in 
which each ambiguous word occurs are used as features. For example, the abstract containing the example phrase in the previous paragraph has been assigned $16 \mathrm{MeSH}$ terms including "M01.060.116.100: Aged", "M01.060.116.100.080: Aged, 80 and over", "D27.505.954.502.119: Anticoagulants" and "G09.188.261.560.150: Blood Coagulation". To our knowledge MeSH terms have not been previously used as a feature for WSD of biomedical documents.

\section{Learning algorithms}

We compared three machine learning algorithms which have previously been shown to be effective for WSD tasks.

The Vector Space Model is a memory-based learning algorithm which was used by [21]. Each occurrence of an ambiguous word is represented as a binary vector in which each position indicates the occurrence/absence of a feature. A single centroid vector is generated for each sense during training. These centroids are compared with the vectors that represent new examples using the cosine metric to compute similarity. The sense assigned to a new example is that of the closest centroid.

The Naive Bayes classifier is based on a probabilistic model which assumes conditional independence of features given the target classification. It calculates the posterior probability that an instance belongs to a particular class given the prior probabilities of the class and the conditional probability of each feature given the target class.

Support Vector Machines have been widely used in classification tasks. SVMs map feature vectors onto a high dimensional space and construct a classifier by searching for the hyperplane in that space that gives the greatest separation between the classes.

We used our own implementation of the Vector Space Model and Weka implementations [27] of the other two algorithms. A linear kernel was used for the Support Vector Machine.

\section{Results}

This system was applied to the entire NLM-WSD data set. Experiments were carried out using each of the three types of features (linguistic, CUI and MeSH) both alone and in combination. Ten-fold cross validation was applied and the figures we report are averaged across all ten runs.

Results from this experiment are shown in Table 1, which lists the performance using combinations of learning algorithm and features. The figure shown for each configuration represents the percentage of instances of ambiguous terms which are correctly disambiguated.
The best performance is obtained using a combination of the linguistic and MeSH features, a pattern observed across all test sets and machine learning algorithms. Although the increase in performance gained from using both the linguistic and MeSH features compared to only the linguistic features is modest, it is statistically significant (Wilcoxon Signed Ranks Test, $p<0.05$ ), as is the difference between using both linguistic and MeSH features compared with using the MeSH features alone $(p<0.01)$.

The Vector Space Model learning algorithm performs significantly better than both Support Vector Machines and Naive Bayes (Wilcoxon Signed Ranks Test, $p<0.01$ ). This pattern is observed regardless of which set of features is used, and it is consistent with the results over SemEval data [21].

Performance using MeSH terms as the only feature is better than using CUIs alone when the Naive Bayes and Support Vector Machine Learning algorithms are used. However, this is reversed for the Vector Space Model. The most likely reason is that the MeSH terms are far more sparse than CUIs (see Discussion section) which hinders this algorithm's performance.

\section{Per-word analysis}

Table 2 shows the results of our best performing system (combination of linguistic and MeSH features using the Vector Space Model learning algorithm). Comparable results for previous supervised systems are also reported where available. To allow direct comparison the results from Joshi et. al. [16] are computed using global parameter setting (see WSD of Biomedical Text section). An equivalent set of results are not available for Liu et al. [14]. Results from Humphrey et al. [20] are also omitted since their system was evaluated against only some of the instances of each term. The MFS baseline for each term is shown in the leftmost column.

The performance of Leroy and Rindflesch's system is always lower than the best result for each word. The systems reported by Joshi et al. [16] and McInnes et al. [17] are better than, or the same as, all other systems for 23 and 11 words respectively. The system reported here achieves results equal to or better than previously reported systems for 33 terms.

There are five terms for which the performance of our approach is actually lower than the MFS baseline (shown in italics) in Table 2. (In fact, the baseline outperforms all systems for three of these terms.) The performance of our system is within $1 \%$ of the baseline for five of these terms. The remaining pair, "blood pressure" and "failure", are included in the set of problematic words [2]. Examination of the possible senses show that they include pairs with 
Table I: Results from WSD system. Results from WSD system applied to various sections of the NLM-WSD data set using a variety of features and machine learning algorithms. The best results obtained by our system are highlighted in bold font. Results from baseline and previously published approaches are included for comparison.

\begin{tabular}{|c|c|c|c|c|c|c|c|}
\hline \multirow[b]{2}{*}{ Data sets } & \multicolumn{7}{|c|}{ Features } \\
\hline & Linguistic & CUI & $\mathrm{MeSH}$ & $\mathrm{CUI}+\mathrm{MeSH}$ & Linguistic $+M e S H$ & Linguistic+CUI & Linguistic $+\mathrm{MeSH}+\mathrm{CUI}$ \\
\hline & \multicolumn{7}{|c|}{ Vector space model } \\
\hline All words & 87.0 & 85.8 & 81.9 & 86.9 & 87.9 & 87.3 & 87.5 \\
\hline Joshi subset & 82.1 & 79.6 & 76.6 & 81.4 & 83.3 & 82.4 & 82.8 \\
\hline Leroy subset & 77.5 & 74.4 & 70.4 & 75.8 & 79.7 & 78.7 & 78.9 \\
\hline Liu subset & 84.0 & 81.3 & 78.3 & 83.4 & 84.8 & 83.9 & 84.2 \\
\hline \multirow[t]{2}{*}{ Common subset } & 79.1 & 75.1 & 70.4 & 76.9 & 81.1 & 80.0 & 79.7 \\
\hline & \multicolumn{7}{|c|}{ Naive Bayes } \\
\hline All words & 86.4 & 81.2 & 85.7 & 81.1 & 86.4 & 81.7 & 81.8 \\
\hline Joshi subset & 80.9 & 73.4 & 80.1 & 73.7 & 81.1 & 74.1 & 74.5 \\
\hline Leroy subset & 76.9 & 66.1 & 74.6 & 65.9 & 77.5 & 66.5 & 67.2 \\
\hline Liu subset & 82.1 & 75.4 & 81.7 & 75.3 & 82.7 & 76.3 & 76.6 \\
\hline \multirow[t]{2}{*}{ Common subset } & 77.2 & 66.1 & 74.7 & 65.8 & 79.0 & 66.7 & 67.4 \\
\hline & \multicolumn{7}{|c|}{ Support Vector Machine } \\
\hline All words & 85.9 & 83.5 & 85.3 & 84.5 & 86.2 & 85.3 & 86.0 \\
\hline Joshi subset & 80.1 & 76.4 & 79.5 & 78.0 & 80.9 & 79.1 & 80.3 \\
\hline Leroy subset & 75.5 & 69.7 & 72.6 & 72.0 & 77.1 & 74.5 & 76.3 \\
\hline Liu subset & 81.7 & 78.2 & 81.0 & 80.0 & 82.3 & 80.6 & 81.7 \\
\hline \multirow[t]{4}{*}{ Common subset } & 76.3 & 69.8 & 71.6 & 73.0 & 78.1 & 75.1 & 76.9 \\
\hline & & \multicolumn{6}{|c|}{ Previous Approaches } \\
\hline & & \multicolumn{2}{|c|}{ Per-term } & \multicolumn{4}{|c|}{ Global } \\
\hline & MFS baseline & Liu et al. (2004) & Joshi et al. (2005) & Leroy and & Rindflesch (2005) & Joshi et al. (2005) & Mclnnes et. al. (2007) \\
\hline All words & 78.0 & - & - & & - & 86.2 & 85.3 \\
\hline Joshi subset & 66.9 & - & 82.5 & & - & 80.9 & 80.0 \\
\hline Leroy subset & 55.3 & - & 77.4 & & 65.5 & 75.7 & 74.5 \\
\hline Liu subset & 69.9 & 78.0 & 84.9 & & - & 83.3 & 81.9 \\
\hline Common subset & 54.9 & - & 79.8 & & 68.8 & 78.1 & 75.6 \\
\hline
\end{tabular}

similar meanings. For example, the two senses which account for the majority (98\%) of the instances of "blood pressure", which refer to the blood pressure within an organism and the result obtained from measuring this quantity, are very closely related semantically.

\section{Linguistic features}

Our WSD algorithm uses a wider range of linguistic features than previous approaches. Table 3 shows a comparison of each of the three types of linguistic features described in the Features section. Each type of feature is used alone and as part of a pair. Performance of each type of feature used alone is above the relevant MFS baseline, indicating that all three provide useful information for disambiguation. Unigrams are the most effective, followed by salient bigrams with local collocations the least effective. A possible reason for this may lie in the fact that local collocations comprise an extensive feature set, some of which may be redundant or noisy. For all words the pairing of local collocations with unigrams is the most effective with performance only $0.1 \%$ less accurate than combining all three types of linguistic features. However, combining salient bigrams with unigrams generates the best results over each of the four subsets and actually outperforms the combination of all three feature types for two of them. 
Table 2: Per-word performance of best reported systems

\begin{tabular}{|c|c|c|c|c|c|}
\hline & MFS baseline & Leroy and Rindflesch (2005) & Joshi et. al (2005) & Mclnnes et al.(2007) & Reported system \\
\hline adjustment & 62 & 57 & 71 & 70 & 73 \\
\hline association & 100 & - & 100 & 97 & 100 \\
\hline blood pressure & 54 & 46 & 50 & 46 & 53 \\
\hline cold & 86 & - & 90 & 89 & 88 \\
\hline condition & 90 & - & 89 & 89 & 89 \\
\hline culture & 89 & - & 96 & 94 & 95 \\
\hline degree & 63 & 68 & 89 & 79 & 93 \\
\hline depression & 85 & - & 84 & 81 & 86 \\
\hline determination & 79 & - & 85 & 81 & 87 \\
\hline discharge & 74 & - & 95 & 96 & 94 \\
\hline energy & 99 & - & 99 & 99 & 98 \\
\hline evaluation & 50 & 57 & 67 & 73 & 81 \\
\hline extraction & 82 & - & 84 & 86 & 85 \\
\hline failure & 71 & - & 69 & 73 & 73 \\
\hline fat & 71 & - & 84 & 77 & 84 \\
\hline fit & 82 & - & 81 & 87 & 88 \\
\hline fluid & 100 & - & 100 & 99 & 100 \\
\hline frequency & 94 & - & 95 & 94 & 94 \\
\hline ganglion & 93 & - & 95 & 94 & 96 \\
\hline glucose & 91 & - & 92 & 90 & 91 \\
\hline growth & 63 & 62 & 69 & 69 & 72 \\
\hline immunosuppression & 59 & 61 & 79 & 75 & 81 \\
\hline implantation & 81 & - & 93 & 92 & 91 \\
\hline inhibition & 98 & - & 98 & 98 & 98 \\
\hline japanese & 73 & - & 76 & 76 & 77 \\
\hline lead & 71 & - & 88 & 90 & 94 \\
\hline $\operatorname{man}$ & 58 & 80 & 89 & 80 & 86 \\
\hline mole & 83 & - & 94 & 87 & 88 \\
\hline mosaic & 52 & 66 & 87 & 75 & 85 \\
\hline nutrition & 45 & 48 & 52 & 49 & 57 \\
\hline pathology & 85 & - & 85 & 84 & 86 \\
\hline pressure & 96 & - & 91 & 93 & 95 \\
\hline radiation & 61 & 72 & 81 & 81 & 85 \\
\hline reduction & 89 & - & 91 & 92 & 88 \\
\hline repair & 52 & 81 & 87 & 93 & 86 \\
\hline resistance & 97 & - & 97 & 96 & 97 \\
\hline scale & 65 & 84 & 76 & 83 & 88 \\
\hline secretion & 99 & - & 99 & 99 & 99 \\
\hline sensitivity & 49 & 70 & 85 & 92 & 93 \\
\hline sex & 80 & - & 87 & 87 & 87 \\
\hline single & 99 & - & 99 & 98 & 99 \\
\hline strains & 92 & - & 93 & 92 & 93 \\
\hline support & 90 & - & 89 & 91 & 90 \\
\hline surgery & 98 & - & 98 & 94 & 97 \\
\hline transient & 99 & - & 99 & 98 & 99 \\
\hline transport & 93 & - & 94 & 93 & 93 \\
\hline ultrasound & 84 & - & 87 & 85 & 88 \\
\hline variation & 80 & - & 88 & 91 & 94 \\
\hline weight & 47 & 68 & 83 & 79 & 82 \\
\hline white & 49 & 62 & 71 & 74 & 81 \\
\hline
\end{tabular}

\section{Discussion}

Our experiment shows that each of the three types of information (linguistic, CUIs and MeSH) can be used to create a classifier which achieves a reasonable level of disambiguation, since performance exceeds the relevant baseline score. This suggests that each of these can con- tribute to the disambiguation of ambiguous terms in biomedical text. In addition, disambiguation is improved by combining information sources. This is consistent with results over general text. For example, Stevenson and Wilks [10] and Harley and Glennon [28] showed that WSD could benefit from use of several different types of 
Table 3: Contribution of linguistic features. Results from various combinations of types of linguistic features, as described in Features section, combined using Vector Space Model learning algorithm. LC = Local Collocations, SB = Salient Bigrams and U = Unigrams.

\begin{tabular}{rcccccc}
\hline & \multicolumn{6}{c}{ Features } \\
\hline Data sets & LC & SB & U & SB+U & LC+SB & LC+U \\
\hline All words & 79.2 & 82.0 & 86.9 & 85.9 & 86.3 & 86.9 \\
Joshi subset & 72.6 & 74.4 & 81.6 & 82.3 & 81.0 & 82.0 \\
Leroy subset & 66.2 & 66.9 & 76.7 & 77.5 & 76.5 & 77.3 \\
Liu subset & 75.7 & 76.2 & 83.4 & 84.3 & 82.7 & 83.9 \\
Common subset & 69.6 & 77.7 & 79.3 & 79.1 & 77.6 & 78.8
\end{tabular}

information from a dictionary. More recently Specia et al. [11] showed that a combination of information sources could improve disambiguation of Portuguese verbs.

Combining MeSH terms with other features generally improves performance, suggesting that this provides the classifier with information not available from the others. An important difference between MeSH terms and the other features (linguistic and CUIs) is that they are assigned to the entire abstract rather than just individual terms and, as such, provide information about the topic of the abstract which would be hard to derive from more local features. This can be seen in the example usage of "adjustment" in the Features section above. The abstract in which this term is used discusses the treatment of coronary angioplasty using heparin, an anticoagulant. This abstract does not include the term "anticoagulant" but is assigned the MeSH term "D27.505.954.502.119: Anticoagulants". It would be difficult to determine that this abstract discusses anticoagulants using only the kinds of linguistic features used by many WSD systems. However, MeSH terms provide a way of identifying this information. These findings in this study are consistent with results from WSD of general text. For example, Agirre and Martinez [21] observed a small improvement when domain information was used as additional information in their WSD system.

Unlike MeSH terms, the inclusion of CUIs as features does not always improve performance and, in several cases, causes it to fall. This is consistent with McInnes et al. [17] who concluded that CUIs were a useful information source for disambiguation of biomedical text but that they were not as robust as one type of linguistic information (unigrams) which they had used for a previous system. However, in some ways this result is surprising since CUIs are derived from UMLS, a resource which contains all the information in the MeSH hierarchy (the MeSH hierarchy is a subset of UMLS). The most likely reason for this is that our CUI assignment, provided by MetaMap, is automatic.
MetaMap does not attempt to disambiguate terms which map onto more than one UMLS concept so this CUI assignment is noisy.

Differences between CUIs and MeSH terms were explored further through an analysis of their distribution in the NLM-WSD corpus. A first observation is that CUIs are far more frequent than MeSH terms. On average 489 CUIs are assigned to each abstract in the NLM-WSD data set and only 13.8 MeSH terms. Two measures were used to determine how well CUIs and MeSH terms indicate the meaning of an ambiguous term. The first of these, entropy, is a measure of uncertainly [29]. Lower entropy values indicate there is less variation in the meanings of abstracts to which a particular CUI or MeSH term is assigned. Entropy is computed using equation 1 where $F$ is a feature (such as CUI or MeSH term), $n$ is the total number of senses and $p_{i}$ is the probability that an instance of that feature is assigned to an abstract which has been assigned a particular sense ( $i$ ).

$$
\operatorname{Entropy}(F)=\sum_{i=1}^{n}-p_{i} \log _{2} p_{i}
$$

The entropy of each feature (CUI or MeSH term) is computed and averaged across all terms in the data set. For CUIs this figure is 0.389 , significantly higher than the equivalent figure for MeSH terms, 0.275 (Wilcoxon Signed Ranks Test, $p<0.01$ ). The higher entropy figure indicates that CUIs provide less information about the sense of an ambiguous term than MeSH terms.

An additional metric, Information Gain [30], is based on entropy and provides a measure of how useful a feature is to classify the data. It can be used to determine how accurately a CUI or MeSH term indicates the sense being used in an abstract to which it has been assigned. Information Gain is computed using the formula shown in equation 2 where $C$ is a collection of texts and $V$ alues $(F)$ the set of values which the feature $F$ can be assigned. In our case the collection, $C$, is the set of abstracts for a given term in the NLM-WSD collection and $V$ alues $(F)$ is binary, since each CUI and MeSH term is either assigned to a particular abstract or not.

$$
\text { Information Gain }(C, F)=\operatorname{Entrophy}(C)-\sum_{v \in \text { Values }(F)} \frac{\left|C_{v}\right|}{|C|} \operatorname{Entropy}\left(C_{v}\right)
$$

The average Information Gain score for all features which apply to a term was computed. The average of this figure across all terms is 0.014 for CUIs and significantly higher (0.017) for MeSH terms (Wilcoxon Signed Ranks Test, $p<$ 0.01 ). This indicates that MeSH terms provide more useful information for sense classification than CUIs. 
The methodology adopted in this study has been to evaluate and compare a variety of types of information which may be useful for the disambiguation of biomedical terms. Each of these sources are readily available: linguistic features can be extracted directly from text, MeSH terms are available for many MEDLINE entries while CUIs can be generated by MetaMap. The study does not directly compare the usefulness or value of the MeSH hierarchy against UMLS since we are using manually assigned MeSH terms and CUIs from UMLS which are automatically generated. We do not have access to a reliable assignment of CUIs to text; if we had WSD would not be necessary since the senses used in the NLM-WSD corpus are effectively CUIs. However, our study suggests that using linguistic features is a better strategy for WSD of biomedical terms, confirming previous results [17]; in addition, there is nothing to be gained from combining CUIs with linguistic features. On the other hand, our study also shows that $\mathrm{MeSH}$ terms can improve disambiguation performance and should be used if available (such as disambiguation of terms in MEDLINE abstracts). While the benefit provided by MeSH terms is statistically significant, it is quite small and not crucial for disambiguation of biomedical text.

\section{Conclusion}

This paper has compared a variety of information sources for WSD of ambiguous biomedical terms and reported results which exceed the performance of previously published approaches. We found that the most accurate results can be achieved using a combination of linguistic features commonly used for WSD of general text and manually assigned MeSH terms. While CUIs are a useful source of information for disambiguation, they do not improve the performance of the best system configuration, i.e. when used in addition to linguistic features and MeSH terms. This may be because our approach uses manually assigned MeSH terms while the CUIs are obtained automatically using MetaMap. Analysis of the information gain afforded by automatically assigned CUIs versus manually assigned MeSH terms for the sense classification task confirms that the MeSH terms do indeed supply more information.

The linguistic information used in this paper comprises a wide variety of features including unigrams, local collocational features and salient bigrams. When these feature types are considered singly unigrams are the most effective, while unigrams together with local collocations are the most effective pair. We have not explored the contribution of individual collocational features, however, and this is a topic for further work. In addition, our approach does not make use of the fact that MeSH terms are organised into a hierarchy. It would be interesting to discover whether this information could be used to improve WSD performance.

Others, for example [31], have developed techniques to make use of hierarchical information in WordNet for WSD which could be adapted to MeSH.

\section{Competing interests}

The authors declare that they have no competing interests.

\section{Authors' contributions}

DM and YG developed the WSD system used in this study. YG generated all experimental results. MS carried out analysis of previous approaches and, together with RG, conceived the study and co-ordinated its design and the drafting of this manuscript. All authors read and approved the final manuscript.

\section{Acknowledgements}

The authors are grateful for the feedback provided by three anonymous reviewers of an earlier version of this paper which appeared at the BioNLP workshop at ACL 2008. We also thank Ted Pedersen and Bridget McInnes for providing detailed performance of their WSD systems. This work was funded by the UK Engineering and Physical Sciences Research Council (grant number EP/E004350/I) and the Australian Research Council (grant number DP0663879).

This article has been published as part of BMC Bioinformatics Volume 9 Supplement II, 2008: Proceedings of the BioNLP 08 ACL Workshop: Themes in biomedical language processing. The full contents of the supplement are available online at http://www.biomedcentral.com/ $|47|-2105 / 9$ ? issue=SI I

\section{References}

I. Humphreys L, Lindberg D, Schoolman H, Barnett G: The Unified Medical Language System: An Informatics Research Collaboration. Journal of the American Medical Informatics Association 1998, I(5): I-II.

2. Weeber M, Mork J, Aronson A: Developing a Test Collection for Biomedical Word Sense Disambiguation. Proceedings of AMAI Symposium, Washington, DC 2001:746-50.

3. Aronson A, Bodenreider $\mathrm{O}$, Chang $\mathrm{H}$, Humphrey S, Mork J, Nelson $\mathrm{S}$, Rindflesch T, Wilbur W: The NLM Indexing Initiative. Proceedings of the AMIA Symposium, Los Angeles, CA 2000.

4. Friedman C: A Broad Coverage Natural Language Processing System. Proceedings of the AMIA Symposium, Los Angeles, CA 2000:270-274.

5. Weeber M, Klein H, Aronson A: Text-based Discovery in Biomedicine: the Architecture of the DAD-system. Proceedings of the AMIA Symposium, Los Angeles, CA 2000:903-907.

6. Swanson D, Smalheiser N: An interactive system for finding complementary literatures: a Stimulus to Scientific Discovery. Artificial Intelligence 1997, 91:183-203.

7. Ide N, Véronis J: Introduction to the Special Issue on Word Sense Disambiguation: The State of the Art. Computational Linguistics 1998, 24: I-40.

8. Agirre E, Edmonds P, (Eds): Word Sense Disambiguation: Algorithms and Applications Text, Speech and Language Technology, Springer; 2007.

9. Schuemie M, Kors J, Mons B: Word Sense Disambiguation in the Biomedical Domain: An Overview. Journal of Computational Biology 2005, I 2(5):554-565.

10. Stevenson M, Wilks Y: The Interaction of Knowledge Sources in Word Sense Disambiguation. Computational Linguistics 2001, 27(3):32l-350.

II. Specia L, Stevenson M, Nunes G: Learning Expressive Models for Word Sense Disambiguation. Proceedings of 45th Meeting of the 
Association for Computational Linguistics (ACL 2007), Prague, Czech Republic 2007:4I-48.

12. Agirre E, Marquez L, Wicentowski R, (Eds): SemEval 2007: Proceedings of the 4th International Workshop on Semantic Evaluations, Prague, Czech Republic 2007.

13. Widdows D, Peters S, Cedernerg S, Chan C, Steffen D, Buitelaar P: Unsupervised Monolingual and Bilingual Word-sense Disambiguation of Medical Documents using UMLS. Workshop on "Natural Langauge Processing in Biomedicine" at ACL 2003, Sapporo, Japan 2003:9-16.

14. Liu H, Teller V, Friedman C: A Multi-aspect Comparison Study of Supervised Word Sense Disambiguation. Journal of the American Medical Informatics Association 2004, I I (4):320-33I.

15. Leroy G, Rindflesch T: Effects of Information and Machine Learning algorithms on Word Sense Disambiguation with small datasets. International Journal of Medical Informatics 2005, 74(7-8):573-585.

16. Joshi M, Pedersen T, Maclin R: A Comparative Study of Support Vector Machines Applied to the Word Sense Disambiguation Problem for the Medical Domain. Proceedings of the Second Indian Conference on Artificial Intelligence (IICAI-05), Pune, India 2005:3449-3468.

17. Mclnnes B, Pedersen T, Carlis J: Using UMLS Concept Unique Identifiers (CUIs) for Word Sense Disambiguation in the Biomedical Domain. Proceedings of the Annual Symposium of the American Medical Informatics Association, Chicago, IL 2007:533-537.

18. McCarthy D, Koeling R, Weeds J, Carroll J: Finding Predominant Senses in Untagged Text. Proceedings of the 42nd Annual Meeting of the Association for Computational Lingusitics (ACL-2004), Barcelona, Spain 2004:280-287.

19. Aronson A: Effective mapping of biomedical text to the UMLS Metathesaurus: the MetaMap program. Proceedings of the American Medical Informatics Association (AMIA) 200I:I7-2I.

20. Humphrey S, Rogers W, Kilicoglu H, Demner-Fushman D, Rindflesch $T$ : Word Sense Disambiguation by selecting the best semantic type based on Journal Descriptor Indexing: Preliminary experiment. Journal of the American Society for Information Science and Technology 2006, 57(5):96-II3.

21. Agirre E, Martínez D: The Basque Country University system English and Basque tasks. Senseval-3: Third International Workshop on the Evaluation of Systems for the Semantic Analysis of Text. Barcelona, Spain 2004:44-48.

22. Mihalcea R, Chklovski T, Kilgarriff A: The Senseval-3 English Lexical Sample Task. Proceedings of Senseval-3: The Third International Workshop on the Evaluation of Systems for the Semantic Analysis of Text, Barcelona, Spain 2004.

23. Ratnaparkhi A: A Maximum Entropy Model for Part-of-Speech Tagging. Proceedings of the Conference on Empirical Methods in Natural Language Processing, Philadelphia, PA 1996:133-142.

24. Pedersen T: A Decision Tree of Bigrams is an Accurate Predictor of Word Sense. Proceedings of the Second Meeting of the North American Chapter of the Association for Computational Linguistics (NAACL-OI), Pittsburgh, PA 200I:79-86.

25. Stevenson M, Guo Y, Gaizauskas R, Martinez D: Knowledge Sources for Word Sense Disambiguation of Biomedica Text. Proceedings of the Workshop on Current Trends in Biomedical Natural Language Processing at ACL 2008 2008:80-87.

26. Nelson S, Powell T, Humphreys B: The Unified Medical Language System (UMLS) Project. In Encyclopedia of Library and Information Science Edited by: Kent A, Hall CM. Marcel Dekker, Inc; 2002.

27. Witten I, Frank E: Data Mining: Practical machine learning tools and techniques San Francisco: Morgan Kaufmann; 2005.

28. Harley A, Glennon D: Sense Tagging in Action: Combining Different Tests with Additive Weights. Proceedings of the SIGLEX Workshop "Tagging Text with Lexical Semantics", Washington, DC 1997:74-78.

29. Manning C, Schütze $\mathrm{H}$ : Foundations of Statistical Natural Language Processing Cambridge, MA: MIT Press; 1999.

30. Mitchell T: Machine Learning New York: McGraw-Hill; 1997.

31. Budanitsky A, Hirst G: Evaluating WordNet-based measures of semantic distance. Computational Linguistics 2006, 32:13-47.
Publish with Bio Med Central and every scientist can read your work free of charge

"BioMed Central will be the most significant development for disseminating the results of biomedical research in our lifetime. "

Sir Paul Nurse, Cancer Research UK

Your research papers will be:

- available free of charge to the entire biomedical community

- peer reviewed and published immediately upon acceptance

- cited in PubMed and archived on PubMed Central

- yours - you keep the copyright
BioMedcentral 